\title{
REFLECTION ARRANGEMENTS ARE HEREDITARILY FREE
}

\author{
TORSTEN HOGE AND GERHARD RÖHRLE
}

\begin{abstract}
Suppose that $W$ is a finite, unitary, reflection group acting on the complex vector space $V$. Let $\mathcal{A}=\mathcal{A}(W)$ be the associated hyperplane arrangement of $W$. Terao has shown that each such reflection arrangement $\mathcal{A}$ is free. Let $L(\mathcal{A})$ be the intersection lattice of $\mathcal{A}$. For a subspace $X$ in $L(\mathcal{A})$ we have the restricted arrangement $\mathcal{A}^{X}$ in $X$ by means of restricting hyperplanes from $\mathcal{A}$ to $X$. In 1992, Orlik and Terao conjectured that each such restriction is again free. In this note we settle the outstanding cases confirming the conjecture.

In 1992, Orlik and Terao also conjectured that every reflection arrangement is hereditarily inductively free. In contrast, this stronger conjecture is false however; we give two counterexamples.
\end{abstract}

\section{INTRODUCTION}

Suppose that $W$ is a finite, unitary, reflection group acting on the complex vector space $V$. Let $\mathcal{A}=(\mathcal{A}, V)=(\mathcal{A}(W), V)$ be the associated hyperplane arrangement of $W$. Terao [Ter80] has shown that each reflection arrangement $\mathcal{A}$ is free and that the multiset of exponents $\exp \mathcal{A}$ of $\mathcal{A}$ is given by the coexponents of $W$ (see also [OT92, §6]).

Let $L(\mathcal{A})$ be the intersection lattice of $\mathcal{A}$. For a subspace $X \in L(\mathcal{A})$ we consider the restricted arrangement $\mathcal{A}^{X}=\left(\mathcal{A}^{X}, X\right)$ in $X$ by means of restricting hyperplanes from $\mathcal{A}$ to $X$. In 1992, Orlik and Terao [OT92, Conj. 6.90] conjectured that each such restriction is again a free arrangement. Free arrangement with this property are called hereditarily free, [OT92, Def. 4.140]. In this note we settle the outstanding cases confirming the conjecture:

Theorem 1.1. For $W$ a finite complex reflection group, the reflection arrangement $\mathcal{A}=$ $\mathcal{A}(W)$ is hereditarily free.

Note that in general the restriction of a free arrangement need not be free again (cf. [OT92, Ex. 4.141]).

Thanks to [OT92, Prop. 4.28], Theorem 1.1 reduces readily to the case when $\mathcal{A}=\mathcal{A}(W)$ is irreducible.

In [OT92, Prop. 6.73, Prop. 6.77, Cor. 6.86], Orlik and Terao proved that each restricted arrangement $\mathcal{A}^{X}$ is again free provided that $W$ is a symmetric group or a monomial group $G(r, p, \ell)$. The case when $W$ is a cyclic group is trivial. This settles Theorem 1.1 for each of the infinite series of complex reflection groups.

2010 Mathematics Subject Classification. Primary 20F55; Secondary 52B30, 52C35, 14N20 13N15.

Key words and phrases. Complex reflection groups, Freeness of restrictions of reflection arrangements. 
Furthermore, in a case-by-case study, Orlik and Terao showed in [OT93] that $\mathcal{A}^{X}$ is free when $W$ is a Coxeter group. In case $W$ is a Weyl group, Douglass [Dou99] gave a uniform proof of this fact using an elegant conceptual Lie theoretic argument.

Moreover, for any hyperplane arrangement $\mathcal{A}$, it is known that $\mathcal{A}^{X}$ is free in case $\operatorname{dim} X=1$, [OT92, Def. 4.7; Prop. 4.27], as well as when $\operatorname{dim} X=2$ [OT92, Ex. 4.20].

For the exceptional complex reflection groups, Orlik and Terao checked that in each instance when $\operatorname{dim} X=3$, the restriction $\mathcal{A}^{X}$ is again free [OT92, App. D].

In this note, we settle the remaining instances in the exceptional groups. There are only four instances when the freeness of $\mathcal{A}^{X}$ still needs to be checked: Either $W=G_{33}$ and $X$ is a hyperplane in $V$ (there is only one class of hyperplanes and here $\operatorname{dim} X=4$ ), or else $W=G_{34}$ and $X$ is a hyperplane in $V$ (there is only one class of hyperplanes and here $\operatorname{dim} X=5$ ), or $X \in L(\mathcal{A})$ belongs to one of two classes of 4-dimensional subspaces in $V$.

Our proof of these remaining cases for Theorem 1.1 is computational. First we use the functionality for complex reflection groups provided by the CHEVIE package in GAP (and some GAP code by J. Michel) (see [S+97] and [GHL ${ }^{+}$96]) in order to obtain explicit linear functionals $\alpha$ defining the hyperplanes ker $\alpha$ of the underlying reflection arrangement $\mathcal{A}(W)$. These then allow us to implement the module of derivations $D(\alpha)$ associated with $\alpha$ in the SINGULAR computer algebra system (cf. [GPS09]). Then the module theoretic functionality of SINGULAR is used to show that the modules of derivations in question $D\left(\mathcal{A}^{X}\right)$ are free.

While our calculations (combined with the existing known instances of the conjecture of Orlik and Terao) do provide a proof of Theorem 1.1, it would nevertheless be very desirable to have a uniform, conceptual proof free of case-by-case considerations and free of computer calculations. A conceptual proof is only known in case of Weyl groups [Dou99].

The notion of freeness was introduced by Saito in his seminal work [S80]. Questions of freeness play a central role in the understanding of arrangements (see [OT92, §4], [Ter80]). In current research they are still of key importance; for instance in form of inductively free arrangements, e.g., see [BC12] or [HR12], or in the context of multiarrangements, e.g., see [Sch12].

In the next section, we recall the required notation and facts about freeness of hyperplane arrangements and reflection arrangements from [OT92, §4, §6]. We settle the outstanding cases of Orlik and Terao's conjecture, completing the proof of Theorem 1.1 in Section 3.

For general information about arrangements and reflection groups, we refer the reader to [OT92] and [Bou68].

\section{Preliminaries}

2.1. Hyperplane Arrangements. Let $V=\mathbb{C}^{\ell}$ be an $\ell$-dimensional complex vector space. A hyperplane arrangement is a pair $(\mathcal{A}, V)$, where $\mathcal{A}$ is a finite collection of hyperplanes in $V$. Frequently, we simply write $\mathcal{A}$ in place of $(\mathcal{A}, V)$. The lattice of $\mathcal{A}, L(\mathcal{A})$, is the set of subspaces of $V$ of the form $H_{1} \cap \cdots \cap H_{n}$, where $\left\{H_{1}, \ldots, H_{n}\right\}$ is a subset of $\mathcal{A}$. 
For $X \in L(\mathcal{A})$, we have two associated arrangements, firstly the subarrangement $\mathcal{A}_{X}:=$ $\{H \in \mathcal{A} \mid X \subseteq H\}$ of $\mathcal{A}$ and secondly, the restriction of $\mathcal{A}$ to $X,\left(\mathcal{A}^{X}, X\right)$, where $\mathcal{A}^{X}:=$ $\left\{X \cap H \mid H \in \mathcal{A} \backslash \mathcal{A}_{X}\right\}$.

Let $S=S\left(V^{*}\right)$ be the symmetric algebra of the dual space $V^{*}$ of $V$. If $x_{1}, \ldots, x_{\ell}$ is a basis of $V^{*}$, then we identify $S$ with the polynomial ring $\mathbb{C}\left[x_{1}, \ldots, x_{\ell}\right]$. Letting $S_{p}$ denote the $\mathbb{C}$-subspace of $S$ consisting of the homogeneous polynomials of degree $p$ (along with 0 ), we see that $S$ is naturally $\mathbb{Z}$-graded: $S=\bigoplus_{p \in \mathbb{Z}} S_{p}$, where $S_{p}=0$ in case $p<0$.

Let $\operatorname{Der}(S)$ be the $S$-module of $\mathbb{C}$-derivations of $S$, where the $S$-module structure is defined as follows: For $f \in S$ and $\theta_{1}, \theta_{2} \in \operatorname{Der}(S)$, let $f \theta_{1}, \theta_{1}+\theta_{2} \in \operatorname{Der}(S)$ be defined by $\left(f \theta_{1}\right)(g):=$ $f \cdot \theta_{1}(g)$ and $\left(\theta_{1}+\theta_{2}\right)(g)=\theta_{1}(g)+\theta_{2}(g)$ for $g \in S$. For $i=1, \ldots, \ell$, let $D_{i}:=\partial / \partial x_{i}$ be the usual derivation of $S$. Then $D_{1}, \ldots, D_{\ell}$ is a $\mathbb{C}$-basis of $\operatorname{Der}(S)$. We say that $\theta \in \operatorname{Der}(S)$ is homogeneous of polynomial degree $p$ provided $\theta=\sum_{i=1}^{\ell} f_{i} D_{i}$, where $f_{i} \in S_{p}$ for each $1 \leq i \leq \ell$. In this case we write $\operatorname{pdeg} \theta=p$. Let $\operatorname{Der}(S)_{p}$ be the $\mathbb{C}$-subspace of $\operatorname{Der}(S)$ consisting of all homogeneous derivations of polynomial degree $p$. Then $\operatorname{Der}(S)$ is a graded $S$-module: $\operatorname{Der}(S)=\bigoplus_{p \in \mathbb{Z}} \operatorname{Der}(S)_{p}$. For instance, the Euler derivation $\theta_{E}:=\sum_{i=1}^{\ell} x_{i} D_{i}$ is homogeneous of polynomial degree 1 (cf. [OT92, Def. 4.7]).

Following [OT92, Def. 4.4], for $f \in S$, we define the $S$-submodule $D(f)$ of $\operatorname{Der}(S)$ by

$$
D(f):=\{\theta \in \operatorname{Der}(S) \mid \theta(f) \in f S\} .
$$

Let $\mathcal{A}$ be an arrangement in $V$. Then for $H \in \mathcal{A}$ we fix $\alpha_{H} \in V^{*}$ with $H=\operatorname{ker}\left(\alpha_{H}\right)$. The defining polynomial $Q(\mathcal{A})$ of $\mathcal{A}$ is given by $Q(\mathcal{A}):=\prod_{H \in \mathcal{A}} \alpha_{H} \in S$.

The module of $\mathcal{A}$-derivations of $\mathcal{A}$ is defined by

$$
D(\mathcal{A}):=D(Q(\mathcal{A})) .
$$

Note that for any arrangement $\mathcal{A}$ we have that $\theta_{E} \in D(\mathcal{A})$ (cf. [OT92, Def. 4.7]). We say that $\mathcal{A}$ is free if the module of $\mathcal{A}$-derivations $D(\mathcal{A})$ is a free $S$-module.

With the $\mathbb{Z}$-grading of $\operatorname{Der}(S)$, the module of $\mathcal{A}$-derivations becomes a graded $S$-module $D(\mathcal{A})=\bigoplus_{p \in \mathbb{Z}} D(\mathcal{A})_{p}$, where $D(\mathcal{A})_{p}=D(\mathcal{A}) \cap \operatorname{Der}(S)_{p}[$ OT92, Prop. 4.10]. If $\mathcal{A}$ is a free arrangement, then the $S$-module $D(\mathcal{A})$ admits a basis of $\ell$ homogeneous derivations, say $\theta_{1}, \ldots, \theta_{\ell}$ [OT92, Prop. 4.18]. While the $\theta_{i}$ 's are not unique, their polynomial degrees pdeg $\theta_{i}$ are unique (up to ordering). This multiset is the set of exponents of the free arrangement $\mathcal{A}$ and is denoted by $\exp \mathcal{A}$.

An important theorem for free arrangements $\mathcal{A}$ states that the Poincaré polynomial $\pi(\mathcal{A}, t)$ of the lattice $L(\mathcal{A})$ (cf. [OT92, §2.3]) factors into linear terms given by the exponents of $\mathcal{A}$ as

$$
\pi(\mathcal{A}, t)=\prod_{i=1}^{\ell}\left(1+b_{i} t\right),
$$

where $\exp \mathcal{A}=\left\{b_{1}, \ldots, b_{\ell}\right\}$ are the exponents of $\mathcal{A}$ [OT92, Thm. 4.137]. This factorization property suggests that freeness of $\mathcal{A}$ only depends on the lattice $L(\mathcal{A})$; this is a basic conjecture due to Terao [OT92, Conj. 4.138]. 
Following [OT92, Def. 4.140], we say that $\mathcal{A}$ is hereditarily free provided $\mathcal{A}^{X}$ is free for every $X \in L(\mathcal{A})$. In general, a free arrangement need not be hereditarily free, thanks to a counterexample due to Edelman and Reiner [OT92, Ex. 4.141].

2.2. Reflection Arrangements. Suppose that $W \subseteq \mathrm{GL}(V)$ is a finite, complex reflection group acting on the complex vector space $V=\mathbb{C}^{\ell}$. The reflection arrangement $\mathcal{A}=\mathcal{A}(W)$ of $W$ in $V$ is the hyperplane arrangement consisting of the reflecting hyperplanes of the elements in $W$ acting as reflections on $V$.

It is known that each reflection arrangement $\mathcal{A}(W)$ is free [Ter80]. In [OT92, Prop. 6.89], Orlik and Terao proved in a case-by-case argument that, for any $X \in L(\mathcal{A})$ with $\operatorname{dim} X=p$, there exist integers $b_{1}^{X}, \ldots, b_{p}^{X}$ such that the Poincaré polynomial of the restriction $\mathcal{A}^{X}$ satisfies the factorization property

$$
\pi\left(\mathcal{A}^{X}, t\right)=\prod_{i=1}^{p}\left(1+b_{i}^{X} t\right) .
$$

Moreover, in all instances when $\mathcal{A}^{X}$ is known to be free, the equality $\exp \mathcal{A}^{X}=\left\{b_{1}^{X}, \ldots, b_{p}^{X}\right\}$ holds. In view of this fact and the aforementioned factorization theorem for free arrangements, [OT92, Thm. 4.137], Orlik and Terao conjecture that every reflection arrangement is hereditarily free (cf. [OT92, Conj. 6.90]). Theorem 1.1 settles this conjecture.

\section{Proof of Theorem 1.1}

As explained in the Introduction, all but four cases of Theorem 1.1 have already been proved. We thus concentrate on the four outstanding incidences in $G_{33}$ and $G_{34}$.

In the case of the restriction of $\mathcal{A}$ to a hyperplane $H$, one is inclined to use the AdditionDeletion Theorem [OT92, Thm. 4.51] in order to derive the freeness of $\mathcal{A}^{H}$. However, in the case of $G_{33}$ and $G_{34}$, the set $\left\{b_{1}^{H}, \ldots, b_{p}^{H}\right\}$ is not a subset of $\exp \mathcal{A}(W)$ (cf. [OT92, Tables C.14, C.17]), and thus this criterion does not apply.

Recall that the defining polynomial $Q(\mathcal{A})=\prod_{H \in \mathcal{A}} \alpha_{H}$ of $\mathcal{A}$ is a product of linear factors. Thanks to [OT92, Prop. 4.8], we have

$$
D(\mathcal{A})=\bigcap_{H \in \mathcal{A}} D\left(\alpha_{H}\right)
$$

Therefore, we may compute $D(\mathcal{A})$ as an intersection of the $S$-modules $D\left(\alpha_{H}\right)$.

The linear factors can be obtained from the CHEVIE package in GAP (see [S+97] and $\left[\mathrm{GHL}^{+}\right.$96]) in the following way:

- $\mathrm{G}:=$ ComplexReflectionGroup (33);

returns the complex reflection group with Shephard-Todd number 33: $G=G_{33}$.

- R:=Reflections (G);

returns the list $R$ of reflections of $G$ (some of which occur more than once).

- A : =MatXPerm $(G, g)$;

returns the representation matrix of $g \in R$ on $V$. 
- BaseMat (A-IdentityMat (1)) [1] ;

returns the linear form $\alpha$ whose kernel is the corresponding hyperplane.

Let $e_{1}, \ldots, e_{\ell} \in V$ be the dual basis of $x_{1}, \ldots, x_{\ell} \in S$. We define a map ${ }^{\vee}: V \rightarrow \operatorname{Der}(S)_{0}$ by $v=\sum_{i=1}^{\ell} \lambda_{i} e_{i} \mapsto v^{\vee}:=\sum_{i=1}^{\ell} \lambda_{i} D_{i} \in \operatorname{Der}(S)_{0}$.

Using this notation, a set of generators of $D(\alpha)$ is given by a basis of the corresponding hyperplane along with the Euler derivation $\theta_{E}$ as follows:

Lemma 3.2. Let $\left\{v_{1}, \ldots, v_{\ell-1}\right\}$ be a $\mathbb{C}$-basis of $\operatorname{ker} \alpha$ for $\alpha \in V^{*} \backslash\{0\}$. Then $\left\{\theta_{E}, v_{1}^{\vee}, \ldots, v_{\ell-1}^{\vee}\right\}$ is an $S$-basis of $D(\alpha)$.

In particular, we have $D(\mathcal{A})_{0}=\bigcap_{H \in \mathcal{A}} H$.

Proof. Note that we have $v_{i}^{\vee}(\alpha)=\alpha\left(v_{i}\right)=0$, and so $v_{i}^{\vee} \in D(\alpha)$, for each $i=1, \ldots, \ell-1$. Let $M:=M\left(\theta_{E}, v_{1}^{\vee}, \ldots, v_{\ell-1}^{\vee}\right)$ be the coefficient matrix associated with $\theta_{E}, v_{1}^{\vee}, \ldots, v_{\ell-1}^{\vee} \in D(\alpha)$, i.e., the entries of $M$ are the coefficients of $\theta_{E}, v_{1}^{\vee}, \ldots, v_{\ell-1}^{\vee}$ in terms of the $D_{i}$ 's (cf. [OT92, Def. 4.11]). Thanks to [OT92, Prop. 4.12], we have $\operatorname{det} M \in \alpha S$. Therefore, once we know that $\operatorname{det} M \neq 0$, we get that $\operatorname{deg}(\operatorname{det} M)=1$, and thus $\operatorname{det} M=\lambda \alpha$ for some $\lambda \in \mathbb{C} \backslash\{0\}$.

Computing $\operatorname{det} M$ by Laplace along the column given by $\theta_{E}$, it is obvious that the determinant does not vanish, since $\left\{v_{1}, \ldots, v_{\ell-1}\right\}$ is linearly independent and therefore at least one $(\ell-1)$-minor of the coefficient-matrix of the $v_{i}^{\vee}$ 's is non-zero. Consequently, $\operatorname{det} M=\lambda \alpha$ for some $\lambda \in \mathbb{C} \backslash\{0\}$. The result now follows from Saito's criterion [OT92, Thm. 4.19].

In order to compute $D\left(\mathcal{A}^{H}\right)$, we require the defining polynomial $Q\left(\mathcal{A}^{H}\right)$ along with its linear factors. The strategy is to insert the equation defining the hyperplane $H$ into the remaining factors of $Q(\mathcal{A})$. Some of the resulting new factors then coincide modulo a scalar, and consequently, the corresponding new hyperplanes are the same. For each hyperplane, we choose only one such factor for the defining polynomial of $\mathcal{A}^{H}$.

Using the explicit data provided by CHEVIE and Lemma 3.2, we can calculate $D\left(\mathcal{A}^{H}\right)$ using equation (3.1) along with the intersect command in SINGULAR (cf. [GPS09]).

The derivations that form a basis of $D\left(\mathcal{A}^{X}\right)$ that we have calculated using SINGULAR in the four cases above are expressions with long and complicated polynomial coefficients, with the exception of the Euler derivation, of course. So they are simply too cumbersome and not particularly enlightening in order to be listed explicitly. The interested reader may find them via the link http://www.ruhr-uni-bochum.de/ffm/Lehrstuehle/Lehrstuhl-VI/hyperplane_arrangement

The following algorithmic method to show that a given arrangement $\mathcal{A}$ is free is proposed by Barakat and Cuntz [BC12, §6.3]. Start with the empty arrangement and successively add hyperplanes. At each step check if the module of derivations given by the corresponding intersections is free. This algorithm only works if $\mathcal{A}$ is inductively free [OT92, Def. 4.53]. In that case there is an ordering of the hyperplanes $H_{1}, H_{2}, \ldots$ such that, for each subarrangement $\mathcal{A}_{i}:=\left\{H_{1}, \ldots, H_{i}\right\}$ of $\mathcal{A}$, the corresponding module of derivations $D\left(\mathcal{A}_{i}\right)$ is free. Unfortunately, neither $\mathcal{A}\left(G_{33}\right)$ nor $\mathcal{A}\left(G_{34}\right)$ is inductively free; see the next paragraph. So it is not possible to employ the algorithm from [BC12] in our case. 
We now show that $\mathcal{A}=\mathcal{A}\left(G_{33}\right)$ is not inductively free; for $G_{34}$ the argument is similar. Our argument depends on the result of our computation that the restricted arrangement $\mathcal{A}^{H}$ is free for $H \in \mathcal{A}$. For, since $\mathcal{A}$ and $\mathcal{A}^{H}$ are free, it follows from [OT92, Prop. 4.57] that the map $q: D(\mathcal{A}) \rightarrow D\left(\mathcal{A}^{H}\right)$ (as defined in [OT92, Prop. 4.45]) is surjective if and only if the subarrangement $\mathcal{A} \backslash\{H\}$ of $\mathcal{A}$ is free. Since the map $q$ is "degree preserving" ([OT92, Prop. 4.44]), it follows from the list of the polynomial degrees of the generators of the free modules $D(\mathcal{A})$ and $D\left(\mathcal{A}^{H}\right)$ (i.e., the exponents of $\mathcal{A}$ and $\mathcal{A}^{H}$ ) given in [OT92, Table C.14] that $q$ is not onto. This in particular shows that $\mathcal{A} \backslash\{H\}$ is not free and so $\mathcal{A}$ is not inductively free. This in particular shows that Orlik and Terao's conjecture that every reflection arrangement is inductively free is false, [OT92, Conj. 6.91]. However, only recently, Barakat and Cuntz showed in [BC12] that every Coxeter arrangement is inductively free. In the forthcoming paper [HR12], we classify all inductively free reflection arrangements.

As indicated above, the computations to settle the four open cases in order to complete the proof of Theorem 1.1 were done using SINGULAR. They were carried out on a 4 x Intel Quad Core Xeon E7340 / 2,4 GHz with 128 GB RAM. The three cases where one has to restrict to a subspace of dimension 4 were computed in less than 2 minutes each. The most elaborate case was to calculate $D\left(\mathcal{A}\left(G_{34}\right)^{H}\right)$. This was computed in 2 days. However, the calculation here might take longer, since SINGULAR uses some random choices in its use of Gröbner bases constructions.

Acknowledgments: We acknowledge support from the DFG-priority program SPP1489 "Algorithmic and Experimental Methods in Algebra, Geometry, and Number Theory".

\section{REFERENCES}

[BC12] M. Barakat and M. Cuntz, Coxeter and crystallographic arrangements are inductively free, Adv. Math 229 (2012), 691-709.

[Bou68] N. Bourbaki, Éléments de mathématique. Groupes et algèbres de Lie. Chapitre IV-VI, Actualités Scientifiques et Industrielles, No. 1337, Hermann, Paris, 1968.

[Dou99] J. M. Douglass, The adjoint representation of a reductive group and hyperplane arrangements, Represent. Theory 3 (1999), 444-456.

$\left[\mathrm{GHL}^{+}\right.$96] M. Geck, G. Hiß, F. Lübeck, G. Malle and G. Pfeiffer, CHEVIE - A system for computing and processing generic character tables, Appl. Algebra Engrg. Comm. Comput. 7 (1996), 175-210.

[GPS09] G.-M. Greuel, G. Pfister and H. Schönemann, Singular 3-1-1, A Computer Algebra System for Polynomial Computations, Centre for Computer Algebra, University of Kaiserslautern, 2009.

[HR12] T. Hoge and G. Röhrle, On inductively free reflection arrangements, preprint 2012, http://arxiv.org/abs/1208.3131

[OT92] P. Orlik and H. Terao, Arrangements of hyperplanes, Springer-Verlag, Berlin, 1992.

[OT93] P. Orlik and H. Terao, Coxeter arrangements are hereditarily free, Tôhoku Math. J. 45 (1993), $369-383$.

[S80] K. Saito, Theory of logarithmic differential forms and logarithmic vector fields, J. Fac. Sci. Univ. Tokyo Sect. IA Math. 27 (1980), no. 2, 265-291.

[S+97] M. Schönert et al., GAP - Groups, Algorithms, and Programming - version 3 release 4, 1997.

[Sch12] M. Schulze, Freeness and multirestriction of hyperplane arrangements, Compositio Math. 148 (2012), 799-806.

[Ter80] H. Terao, Arrangements of hyperplanes and their freeness I, J. Fac. Sci. Univ. Tokyo Sect. IA Math. 27 (1980), 293-320. 
Fakultät für Mathematik, Ruhr-Universität Bochum, D-44780 Bochum, Germany E-mail address: torsten.hoge@rub.de

Fakultät für Mathematik, Ruhr-Universität Bochum, D-44780 Bochum, Germany

E-mail address: gerhard.roehrle@rub.de 